\title{
Reducing Surgical Site Infections in Fractured Neck of Femur Patients: A Closed Loop Audit and Literature Review
}

\author{
Kashif Memon, Randeep Singh Heer, Naeem Raza, Malik Moiz
}

\begin{abstract}
OBJECTIVE: To establish the incidence and identify the risk factors correlated to surgical site infections (SSIs) in geriatric patient having a surgery of fractured neck of femur in a district general hospital (DGH). METHODOLOGY: An initial audit was done for all fractured neck of femur patients who underwent for surgery in a three-month period. Electronic records were checked, and case notes were reviewed. After the initial audit, recommendations were made and the incidence of SSIs was re-audited 16 months later in 2 sessions $\left(2^{\text {nd }}\right.$ and $3^{\text {rd }}$ Audit).

RESULTS: The initial audit in 89 patients showed an infection rate of $8.9 \%$ of which half were deep infections. The mean age was 79 years. Four superficial and 4 deep infections were identified. Patients with superficial infection had, Fixation with DHS, THR, Hemiarthroplasty and IM nailing. Patients with Deep infection had fixation with Dynamic hip screw (DHS), Cannulated hip screw (CHS), Hemiarthroplasty and IM nailing respectively, A Re-audit of 184 patients in 2 sessions ( $2^{\text {nd }}$ and $3^{\text {rd }}$ Audit) showed the infection rate reduced to $0.54 \%$. One patient with infection had hemiarthroplasty, with mean age of patients was 80 years.

CONCLUSION: Multiple techniques can be used to reduce Surgical site infection in fracture neck of femur patients and infection can be reduced to acceptable level such as Senior surgeon operating or Direct supervision, Pre-operative on table skin wash with chlorhexidine soap solution, Use of alcoholic betadine and Chlorhexidine, Two Opsite technique to isolate groin area, Non-braided suture for closure and layered washout, Re application of Chlorhexidine to skin prior to closure.
\end{abstract}

KEY WORDS: Fracture neck of femur; Surgical site infection; Reduction

This article may be cited as: Memon K, Heer RS, Raza N, Moiz M. Reducing Surgical Site Infections in Fractured Neck of Femur Patients: A Closed Loop Audit and Literature Review. J Liaquat Uni Med Health Sci. 2019;18(04):258-61. doi: 10.22442/jlumhs.191840638

\section{INTRODUCTION}

The hip fractures account for over 70,000 admissions to hospital in the UK, costing the NHS and social care $£ 2$ bn / year ${ }^{1}$.

Hip surgery through either fixation or replacement is the most common form of management in elderly patients with fractured neck of femur ${ }^{2}$.

In 2002, it was reported that surgical site infection increases the median length of stay in hospital by fourteen days per patient, almost the double re-hospitalisation rates and raised the healthcare costs by more than $300 \%{ }^{1}$, in another study it was predicted that $1 / 5^{\text {th }}$ of all hospital related infections are surgical site infections, the $2^{\text {nd }}$ most common type of hospital related infection in the USA ${ }^{2}$.

Lau ACK $2014^{3}$ had shown that overall SSI infection rate of $4.3 \%$ in fracture neck of femur patients who underwent hip surgery. The SSI rate observed in another study reported an infection rate of $3.76 \%$ patients with fracture neck of femur ${ }^{4}$.

Surgical site infections (SSI) are a serious complication following fracture NOF surgery and results in increased length of hospital stays and also increases mortality and morbidity.

In general, the elderly population have low physiological reserves, any extra insult in the form of revision surgeries and subsequent infection tend to result in increased morbidity and mortality.

The labrum and cartilage of acetabulum is comparatively a vascular, and biofilm production on the implant, further results to the resistant type of joint infections ${ }^{3}$.

The aim of this closed loop audit and literature review was to find the incidence of surgical site infection in geriatric patients having surgery for fracture neck of femur in a busy DGH and analyse any risk factors correlated with SSI in our patients presenting to Wexham Park Hospital.

\section{METHODOLOGY}

Proforma was created before $1^{\text {st }}$ audit to collect data. All patients age above 65 years undergoing hip surgery for fracture neck of femur in Wexham Park Hospital (WPH), Slough, United Kingdom, between December 2013 to February 2014 were included in this cohort study.

The patients were operated by either; consultants, 
associate specialists or Fellows or trainees under supervision from the same institution. The detection of surgical site infection was conducted by trained nurse. All medical records for patients having primary hip surgery for fracture neck of femur during December 2013 to February 2014 were analysed at thirty days and twelve months post operatively; any recorded infection that matched the USA Centres for Disease Control and Prevention (CDC) benchmark was added in list.

The disease control centre has mentioned no of benchmark for the intent of scrutiny, for the intent of decreasing surgical site infection risk, divided SSIs into: (a) superficial to fascia; (b) deep to fascia; and (c) deep in joint.

Above the fascia surgical site infections is, characterized as infection that resulted during 4 weeks of surgery, affects only structures superficial to fascia, and having either of the following components: clinically pain on touching the wound site or hot to touch, red on inspection or discharging wound; positive microbiology results from an aseptically gained fluid.

Deep surgical site infection is characterised as infection that resulted during 3 months of index surgery and affects the tissues deep to fascial layers.

In deep joint space, infection affecting the joint space or deep to bone. For resemblance of rates of infection across hospitals and measuring rates of SSI within an hospital over different period of times, the Disease control and prevention has suggested the National Nosocomial Infections Surveillance (NNIS) risk index system so that institutes can record cumulative wound infection data.

The NNIS risk index ranges between zero to three points. 1 point is combined to the patient's risk index for any of the three following variables: (a) Surgical procedure that is dirty or contaminated (b) Pre-operative score of 3 and above according to American Society of Anaesthesiologists (ASA) preoperative scoring system; and (c) surgical procedure time more than 75th percentile (determined using the NNIS database).

In our study, as all hip surgeries are considered as clean procedure so the highest NNIS risk index was 2 . After the initial audit, surgical protocol was devised to reduce infection.

\section{Surgical protocol after $1^{\text {st }}$ audit:}

- No wound inspection for 14 days unless clinically indicated

- Senior surgeon operating or Direct supervision

- Pre-operative on table skin wash with chlorhexidine soap solution

- Use of alcoholic betadine and Chlorhexidine

- Two Opsite technique to isolate groin area
- Non-braided suture for closure and layered washout

- $\operatorname{Re}$ application of Chlorhexidine to skin prior to closure

- Absorbable skin sutures with knots buried under skin.

- Use of steri- strips and tincture.

- Mepore / Opsite wound pad for dressing

\section{RESULTS}

An initial audit was conducted from December 2013 February 2014 showing a high rate of SSIs at Wexham Park Hospital.

- Eighty-nine patients identified. Mean age was 79 years. All the patients had got pre-op intravenous antibiotic as per trust policy.

- Eight patients developed surgical site infections.

- Four patients had superficial infection and 4 patients had got deep infection. Out of 89,4 patients with superficial infection, one patient had got DHS, second patient had got THR, third patient had got Hemi Arthroplasty, the last patient had got IM Nailing. Two patients had positive cultures; three patients had skin closure with clips. 3 patients were operated by registrars and one patient was operated by consultant

- Four patients had deep infection, one of the patients had DHS, another patient had Compression hip screw, another patient had Hemi Arthroplasty, and the last patient had IM Nailing. Three of the patients had positive cultures and all patients had skin closure with clips. In this group, again 3 patients were operated by Registrars and one was operated by consultant

In none of cases groin was isolated with separate Opsite. Wounds checked randomly due to concerns of redness around surgical site. Some patients were operated without supervision of consultant.

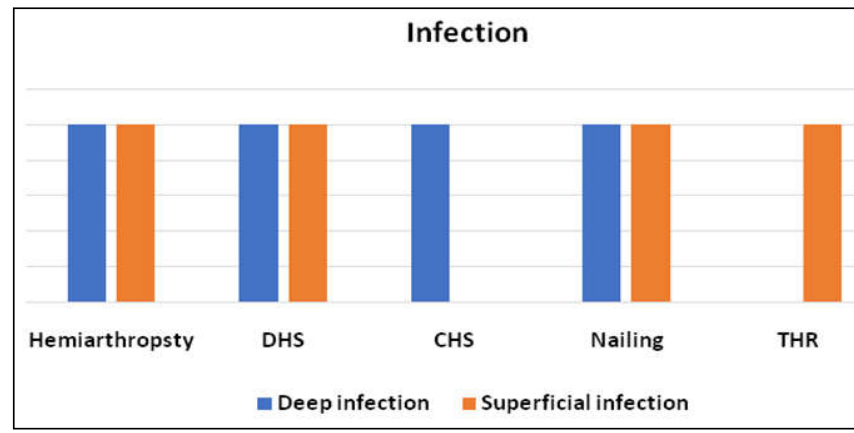

Re-Audit

Second audit performed in December 2016 - February 2017.

In second audit 89 patients identified, no infection, mean age was 79 years, all patients had pre-op 
Intra-venous antibiotics as per trust protocol.

Third audit performed in July 2018 - September 2018. In third audit 95 patients identified, one patient had infection who had hemiarthroplasty procedure done, where wound closure was done with Monocryl and glue. Culture results grew Staph Aureus and Staph Epidermis. Mean age of patient was 81 years. Patient had pre-operative Intravenous antibiotic as per trust policy.

- There has been a reduction in the incidence of SSIs in fractured neck of femur patients between the time periods studied.

- The practices employed may have contributed to this reduction.

- Further study using a longer time period would be able to confirm a definitive reduction in SSIs.

\section{DISCUSSION}

Surgery for fracture neck of femur is mostly performed procedure in elderly patients. Peri-operative treatment of these elderly people should be orthogeriatric and Occupational therapist and physiotherapist in order to optimize medical conditions and avoid post-operative complications, allowing appropriate discharge ${ }^{3}$.

Infection is a serious post-operative complication of surgery for fracture neck of femur, causing increased rates of mortality and morbidity.

We had shown in our audit that initial high rate of infection had been reduced by simple measures. Initial audit shown $8.9 \%$ infection rate half of them was deep infection. Re-audit shows infection rate reduced to $0.54 \%$ with simple measures as mentioned.

The experience level of the operating personnel is an important variable in prevention of infection ${ }^{5}$. The level of experience level of operating personnel was measured by the no of hip surgeries done per year. The operating surgeon can be either supervising or performing the surgical procedure. Further analysis of data did not present any astatically major correlation among the type of surgeon (Hip and knee specialist or upper $\operatorname{limb}$ specialist or spinal specialist). Our results predicted that the experience of operating personnel is more critical than the type of operating personnel. Although, a smaller number of trauma surgeons will result in to a greater number of patients operated per trauma operating personnel per year, theoretically reducing the risk of post-operative infection.

The study by Singh B $2017^{7}$ showed that the wounds closed with sub cut Monocryl had less wound healing issues (average score 0.71) as compared to the wounds closed with clips (average score 1.20).

Magalini $\mathrm{S}$ et $\mathrm{al}^{8}$ concluded is their study chlorhexidine in kits is simple and quick to utilise as compared to PVI and need fewer ancillary substances and has been mentioned in past to decrease surgical site infection in clean contaminated surgery.

\section{CONCLUSION}

Careful patient management for fracture neck of femur is important and likely reduce the chances of surgical site infections that results with increased cost of surgical procedure and morbidity. This study proposes that there are certain amenable causes for SSIs. After establishing those causes and taking appropriate measure, resulting into improved care of SSI prevention in patients with femoral neck fractures. To upgrade the results, simple measures can be employed pre-operatively, intra-operatively and post operatively to reduce chances of surgical site infection.

Ethical permission: No Ethical committee approval needed from NHS REC as this was an audit.

\section{Conflict of interest: Nothing to declare}

Funding: No funding received from any source.

\section{REFERENCES}

1. Whitehouse JD, Friedman ND, Kirkland KB, Richardson WJ, Sexton DJ. The impact of surgical -site infections following orthopaedic surgery at a community hospital and a university hospital: adverse quality of life, excess length of stay, and extra cost. Infect Control Hosp Epidemiol. 2002; 23(4):183-9.

2. Royal College of Physicians (UK). National Hip Fracture Database (NHFD) 2018. Available from: https://www.nhfd.co.uk/files/2018ReportFiles/ NHFD-2018-Annual-Report-v101.pdf.

3. Lau ACK, Neo GH, Lee HC. Risk factors of surgical site infections in hip hemiarthroplasty: a single-institution experience over nine years. Singapore Med J. 2014; 55(10): 535-8.

4. Ji C, Zhu Y, Liu S, Li J, Zhang F, Chen W, et al. Incidence and risk of surgical site infection after adult femoral neck fractures treated by surgery: A retrospective case-control study. Medicine (Baltimore). 2019; 98(11): e14882. doi:10.1097/ MD.0 000000000014882.

5. De Jong L, Klem TMAL, Kuijper TM, Roukema GR. Factors affecting the rate of surgical site infection in patients after hemiarthroplasty of the hip following a fracture of the neck of the femur. Bone Joint J. 2017; 99-B(8): 1088-94. doi: 10.1302/0301-620X.99B8.BJJ-2016-1119.R1. 
6. Noailles T, Brulefert K, Chalopin A, Longis PM, Gouin F. What are the risk factors for postoperative infection after hip hemiarthroplasty? Systematic review of literature. Int Orthop. 2016; 40(9):1843-8. doi: 10.1007/s00264-015-3033-y.

7. Singh B, Bawale R, Sinha S, Gulihar A, Tyler J. Prospective randomised trail comparing the methods of skin closure: subcutaneous sutures versus metal clips. Orthopaedic Proceedings. 2017; 99-B(Suppl 17): 18.

8. Magalini S, Pepe G, Panunzi S, De Gaetano A, Abatini C, Di Giorgio, et al. Observational study on preoperative surgical field disinfection: povidoneiodine and chlorhexidine-alcohol. Eur Rev Med Pharmacol Sci. 2013; 17(24): 3367-75.

AUTHOR AFFILIATION:

Kashif Memon (Corresponding Author)

Speciality Doctor and Shoulder Fellow

Wexham Park and Heather wood Hospital, Frimley NHS Trust

Wexham Road, Slough, United Kingdom.

Email: kashif_nabi@yahoo.com

\section{Randeep Singh Heer}

Foundation Doctor

Wexham Park and Heather wood Hospital, Frimley NHS Trust

Wexham Road, Slough, United Kingdom.

\section{Naeem Raza}

Consultant Hip and knee Surgeon

Wexham Park and Heather wood Hospital, Frimley NHS Trust

Wexham Road, Slough, United Kingdom.

\section{Malik Moiz}

Consultant Hip and knee Surgeon

Wexham Park and Heather wood Hospital, Frimley NHS Trust

Wexham Road, Slough, United Kingdom. 